\section{Vol. 66, No. 29}

In "QuickStats: Age-Adjusted Percentage of Adults Aged $\geq 18$ Years Who Were Never in Pain, in Pain Some Days, or in Pain Most Days or Every Day in the Past 6 Months, by Employment Status - National Health Interview Survey, United States, 2016," on page 796, the caption should have read as follows:

"In 2016, 38.1\% of adults aged $\geq 18$ years never had pain, $\mathbf{4 2 . 6} \%$ had pain on some days, and $\mathbf{1 9 . 4 \%}$ had pain most days or every day in the past 6 months. A higher percentage of adults who were previously employed $(\mathbf{2 9 . 8 \%})$ had pain most days or every day compared with never employed adults (18.6\%) and currently employed adults (15.1\%). Never employed adults $(\mathbf{4 1 . 9 \% )}$ ) and currently employed adults $(\mathbf{4 0 . 4 \% )}$ were more likely to report never having had pain than previously employed adults (31.2\%)."

\section{Vol. 66, No. 33}

In "QuickStats: Percentage of Adults Who Ever Used an E-cigarette and Percentage Who Currently Use E-cigarettes, by Age Group - National Health Interview Survey, United States, 2016," on page 892 , the caption should have read as follows:

"Overall, $\mathbf{1 5 . 3 \%}$ of adults aged $\geq 18$ years had ever used an e-cigarette, and 3.2\% currently used e-cigarettes in 2016. Adults aged 18-24 years were the most likely to have ever used an e-cigarette $(\mathbf{2 3 . 8 \%})$; the percentage declined steadily to $4.4 \%$ among adults aged $\geq 65$ years. Adults aged $18-24$ years (4.7\%) and 25-44 years (4.2\%) were more likely to be current e-cigarette users than adults aged 45-64 years $(\mathbf{2 . 8 \%})$ and those aged $\geq 65$ years $(1.0 \%)$. Across all age groups, fewer than one fourth of adults who had ever used an e-cigarette reported being a current user."

\section{Vol. 66, No. 34}

In "QuickStats: Percentage of Children Aged 6-17 Years Who Wear Glasses or Contact Lenses, by Sex and Age Group National Health Interview Survey, 2016," on page 917, the caption should have read as follows:

"In 2016, the percentage of children aged 6-17 years who wear eyeglasses or contact lenses was higher among girls (35.9\%) compared with boys (29.1\%). Girls aged 6-9 years $(\mathbf{2 0 . 8} \%)$ and $14-17$ years $(\mathbf{5 1 . 4 \%})$ were more likely than boys of the same age group ( $15.4 \%$ and $\mathbf{3 8 . 1 \%}$, respectively) to wear eyeglasses or contact lenses. There was no statistically significant difference by sex for children aged $10-13$ years (35.4\% among girls, 33.7\% among boys). Among both girls and boys, children aged 14-17 years were most likely to wear eyeglasses or contact lenses and children aged 6-9 years were least likely to wear eyeglasses or contact lenses." 